\title{
La pobreza como determinante del consumo doméstico de leña y su efecto en los bosques del Ecuador
}

\author{
Poverty as a determinant of domestic firewood consumption \\ and its effect on the forests of Ecuador
}

\author{
Maribel Caicedo ${ }^{\text {**, María Cristina Vallejo }}{ }^{\text {a }}$, Fernando Carrasco ${ }^{\text {a }}$ \\ *Autor de correspondencia: ${ }^{a}$ Facultad Latinoamericana de Ciencias Sociales, Departamento de Desarrollo, \\ Ambiente y Territorio, La Pradera E7-174 y Av. Diego de Almagro, Quito, Ecuador, \\ tel.: +593984974570, kndymab@hotmail.com
}

\begin{abstract}
SUMMARY
The purpose of this research was to analyze poverty as a determinant factor of domestic consumption of wood and its effect in deforestation in Ecuador. To achieve this, we have processed data from the population and housing census and from household surveys carried out during the last 35 years, forest cover data and a National Forest Assessment. This work makes two contributions. On the one hand, it studies and quantifies deforestation originated by wood consumption, which has been scantily analyzed in Ecuador. On the other hand, it provides a methodological approach that allows a precise accounting of this information through an innovative model of economies of scale, which assumes diminishing marginal consumptions in households. We study the relationship between fuel wood consumption and the state of household poverty through a multivariate analysis that incorporates socioeconomic and demographic variables. With these elements, we show that the largest share of wood consumption does not result from subsistence consumption, but from commercial and productive consumption. For this reason, domestic fuel wood consumption for cooking barely explains deforestation. We have estimated that, from the 49,497 hectares of annual deforested area in the country, 430.6 hectares of forest are affected by the consumption of this resource.
\end{abstract}

Key words: deforestation, economies of scale, multiple correspondence analysis, Ecuador.

\section{RESUMEN}

El propósito de esta investigación fue analizar la pobreza como determinante del consumo doméstico de leña y el efecto que ha generado en la deforestación en territorio ecuatoriano. Para ello, se procesaron datos de los censos de población y vivienda, encuestas de hogares de los últimos 35 años, registros de superficies de bosques y una Evaluación Nacional Forestal. Este trabajo realiza dos aportes. Por un lado, estudia y cuantifica la deforestación originada en el consumo de leña, que ha sido escasamente analizada en el Ecuador; y, por otro lado, aporta con una propuesta metodológica que permite obtener una contabilización precisa de esta información, a través de un modelo original de economías de escala, que asume consumos marginales decrecientes de los hogares. Se estudia la relación entre el consumo de leña y la situación de pobreza de los hogares a partir de una caracterización multivariante, que incorpora variables socioeconómicas y demográficas. Con estos elementos se demuestra que la parte más importante del consumo de leña no tiene lugar por el autoconsumo para subsistencia, sino en el consumo comercial y productivo. Por esta razón, la deforestación que explica el consumo de leña para cocinar es mínima. Se estimó que, de las 47.497 hectáreas deforestadas anualmente en todo el país, 430,6 hectáreas de bosque se ven afectadas por el consumo de este recurso.

Palabras clave: deforestación, economías de escala, análisis de correspondencias múltiple, Ecuador.

\section{INTRODUCCIÓN}

Ecuador es un país rico en recursos naturales por la diversidad de regiones naturales con que cuenta (Costa, Sierra, Amazonía y Galápagos); no obstante, la pérdida de la riqueza forestal es importante. El Ministerio del Ambiente (2015) ha estimado como dato oficial para el país que la tasa anual de cambio de cobertura boscosa en territorio continental es de $-0,37 \%$ para el periodo
2008-2014. Esto corresponde a una deforestación anual promedio de 47.497 ha.

La tasa de deforestación del Ecuador es la quinta más alta dentro de Sudamérica, según cifras de la FAO. Sin embargo, la información forestal es "dispersa, desactualizada, escasa y poco consistente" (Añazco et al. 2010). Datos del periodo previo, por ejemplo, estiman la deforestación entre 80.000 y 350.000 hectáreas por año (Proaño 2005), siendo un rango de variación muy amplio e im- 
preciso, que genera problemas para el fortalecimiento de políticas públicas de conservación medioambiental.

En este estudio interesa profundizar sobre los efectos de la deforestación por el consumo de leña. Aunque el consumo de esta fuente primaria de energía ha ido disminuyendo con la sustitución por gas licuado de petróleo (GLP), su uso es aún común y significativo en algunos países en desarrollo. Más de 2.400 millones de personas, aproximadamente un tercio de la población mundial, dependen todavía del uso tradicional de combustible de madera para cocinar (FAO 2017). Según los censos de población y vivienda del Ecuador, durante las últimas tres décadas y media la incidencia del consumo de leña ha tenido un importante cambio. Del total de hogares registrados en 1982, el 43,7 \% consumió leña; en la actualidad solo el $4,4 \%$ del total de hogares hacen uso de este recurso.

Las cifras disponibles sobre el volumen de consumo de leña, sin embargo, son limitadas; tan solo existen dos reportes, cuyos datos no son comparables porque cubren diferentes escalas. Así, un estudio de la Central Ecuatoriana de Servicios Agrícolas, CESA (1992), calcula un promedio de consumo local de $13,5 \mathrm{~m}^{3}$ por familia al año. El cálculo se basa en la información recolectada en una encuesta realizada en 1991 a tres comunidades de la parroquia de Pilahuin, en la provincia andina de Tungurahua, con el fin de encontrar la cantidad consumida de leña. Este trabajo utiliza un factor de conversión de 12,5 a 14,5 $\mathrm{m}^{3}$ de consumo de leña por familia al año, que permite determinar el promedio de consumo familiar para hogares característicos de la zona, con una media de cinco miembros. Sin embargo, estas cifras parecen sobreestimar el consumo agregado, al ignorar que la estructura y características de los hogares determinan un comportamiento de consumo distinto y, por lo tanto, requieren un tratamiento no lineal.

Por su parte, Proaño (2005) estudia la contribución del bosque en términos de ingresos económicos en madera para leña, que calcula en US\$29.432,96 para un consumo de $4.598,90 \mathrm{~m}^{3}$ al año. Estos valores determinan un factor de conversión de $6,4 \mathrm{~m}^{3}$ de consumo de leña por familia al año, que permiten determinar el consumo a nivel nacional al relacionarlos con los datos de los censos de población y vivienda de 1990 y 2000 (551.000 y 373.428 viviendas, respectivamente). Si se aplican estos factores de conversión, puede comprobarse fácilmente que el primero daría un volumen estimado de consumo de leña de algo más del doble, aunque en estos casos no se toman en cuenta economías de escala en el consumo dentro del hogar.

Con estas cifras se determina que el Ecuador es uno de los países con alta demanda de leña utilizada como combustible en muchos hogares asentados sobre todo en áreas rurales, que circundan bosques y una cantidad no determinada en actividades comerciales o productivas, tales como: restaurantes, asaderos, ladrilleras, panaderías, criaderos de pollos, entre otras. Conforme la FAO (2006a), el consumo que se produce en el sector rural probablemente afecta principalmente y con mayor fuerza a los bosques nativos, amenazando a su vez a las diversas formas de vida y la integridad cultural de quienes dependen directamente de los bosques, que suelen ser poblaciones en situación de pobreza y vulnerabilidad (ancianos, niños y mujeres).

La dependencia de los pobres de zonas rurales respecto a los servicios de los ecosistemas raras veces se mide. Conocer y precisar el volumen de recursos forestales que utilizan los hogares para cocinar sus alimentos o calentarse, así como su impacto sobre los bosques del Ecuador, constituye el propósito central de esta investigación. Sin embargo, el desafío de este artículo en términos analíticos será específicamente evaluar a la pobreza como determinante del uso de leña.

La definición de estos elementos permitirá entender cómo ha ido variando en el tiempo esta dimensión de la deforestación y cómo ha ido relacionándose con la condición socioeconómica de las familias que utilizan leña para cocinar. Desde un punto de vista metodológico, estos resultados contribuirán a la contabilidad de flujos de materiales (Eurostat 2001, Vallejo 2010) a partir de la identificación de un flujo de biomasa orientado a la subsistencia, que generalmente se desestima de las cuentas nacionales oficiales por su escaso peso económico, aunque es un factor fundamental en las condiciones de vida de los hogares que dependen de su consumo. Aquí se argumenta que su contabilización adecuada en cuentas biofísicas, puede constituirse en un elemento central a la hora de definir estrategias apropiadas para la conservación del ambiente y el mejoramiento de las condiciones de vida de las poblaciones que precisan de esta fuente de energía (Sierra et al. 2011).

En efecto, hasta ahora los estudios del sector forestal ecuatoriano han sido escasamente utilizados como insumo del Estado para la toma de decisiones en la implantación de políticas públicas. Algunos han servido como fuentes de información descriptiva sobre la situación forestal (CESA 1992, Wunder 1996, Proaño 2005), otros tienen algunos elementos que pueden contribuir en términos de política pública (McKenzie 1994, Añasco et al. 2010, FAO 2012). Sin embargo, el punto en común de todos estos trabajos es la ausencia de cifras consistentes de la deforestación originada en el consumo de leña.

En tal contexto, es relevante preguntarse si ¿son los más pobres el origen de la presión ambiental sobre los bosques del Ecuador, debido a su consumo de leña? A partir de las investigaciones existentes, parecería que una hipótesis plausible como punto de partida es que la proporción más importante del consumo de leña no se produce en el autoconsumo para subsistencia de los más pobres sino en el consumo para negocios o actividades productivas. Para responder a esta cuestión, en este trabajo se desarrolla un modelo de economías de escala con consumos marginales decrecientes para el uso de leña de los hogares, el cual se relaciona con las condiciones socioeconómicas de las familias que utilizan la leña para cocinar. Una debilidad que persiste en trabajos de esta naturaleza es la escasez de información actualizada. Las cifras de base más recientes 
que existen por el momento corresponden al estudio levantado por FAO (2012), cuyo análisis puede realizarse solo a partir de proyecciones estadísticamente consistentes.

\section{MÉTODOS}

Área de estudio e información forestal. El estudio se realizó cubriendo todo el territorio ecuatoriano $\left(256.370 \mathrm{~km}^{2}\right)$. En 1990, Ecuador poseía 14.587.771 hectáreas de bosque nativo; para 2014 esta extensión se redujo a 12.753.387 hectáreas, con una tasa anual promedio de deforestación de $-0,37 \%$. Durante este periodo, cada año se deforestaron en promedio $47.497 \mathrm{ha}$.

Cada región del Ecuador continental está cubierta por distintos tipos de bosques, cuyas características dependen principalmente del clima y del tipo de suelo. Los datos de la superficie cubierta con diferentes tipos de bosques se basan en nueve estratos que representan una remanencia aproximada de 11,3 millones de hectáreas de bosque nativo para el año 2013. Esta información está actualizada y homogeneizada con el Mapa de Ecosistemas (MAE 2013) y Mapa de Deforestación Histórica (MAE 2010), según información obtenida por fotografías aéreas e imágenes satelitales MODIS y Landsat. Se identifican tres grupos de bosques:

- Bosques secos: Seco Andino, Seco Pluvioestacional.

- Bosques andinos: Siempre Verde Andino Montano, Siempre Verde Andino de Pie de Monte, Siempre Verde Andino de la Ceja Andina.

- Bosques húmedos: Siempre Verde de Tierras Bajas de la Amazonía, Siempre Verde de Tierras Bajas del Choco, Manglar, Moretal.

Estos tipos de bosque, en volumen comercial de madera, equivalen a $241,65 \mathrm{~m}^{3} \mathrm{ha}^{-1}$ (promedio ponderado), que en volumen total de bosque se estiman en $2.746 .340 .287 \mathrm{~m}^{3}$ (MAE 2015).

Datos. En esta investigación se trabajó con la información oficial reportada por las instituciones con mayor incidencia en el tema, como son: el Ministerio del Ambiente del Ecuador (MAE), la Organización de las Naciones Unidas para la Alimentación y la Agricultura (FAO) y el Instituto Nacional de Estadística y Censos (INEC).

La información del INEC, con respecto a la evolución del consumo de leña a nivel de hogares, está disponible en los censos y encuestas oficiales de los últimos 35 años. Se emplean cifras de los tipos de combustibles usados en el hogar, así como información de las condiciones sociodemográficas, de la vivienda, y un módulo muy detallado de características de la población. Las encuestas tienen metodologías de levantamiento de datos y diseños de muestras, que permiten obtener estimaciones de indicadores socioeconómicos con un alto grado de precisión y confiabilidad. Las encuestas de empleo tienen dominios provinciales, excepto para la Amazonía, mientras que las encuestas de condiciones de vida permiten realizar estimaciones a nivel de las regiones naturales del país. Se utilizan las siguientes fuentes:

- Censos de población y vivienda (CPV) 1982, 1990, 2001 y 2010.

- Encuestas de condiciones de vida (ECV) 1995, 1998, 1999, 2005-2006 y 2013-2014.

- Encuestas de empleo y desempleo (ENEMDU), cuarta ronda de los años 2007, 2008, 2009, 2010, 2011, 2012, 2013, 2014, 2015, 2016 y 2017.

La fuente principal de cifras sobre deforestación es el estudio piloto efectuado en la provincia amazónica de Sucumbíos (FAO 2012). A partir de esta evaluación forestal se obtuvieron diferentes tipos de consumidores clasificados según sus economías de escala. Estos grupos han sido proyectados en base a los censos y encuestas nacionales, y sus resultados han sido validados estadísticamente. La metodología empleada por FAO (2012) para el levantamiento de información da cuenta de cómo se utiliza la leña mediante la relación gente-bosque y posee un diseño probabilístico que garantiza resultados muy confiables. La encuesta de Sucumbíos, que fue realizada en el año 2012, cuenta con una muestra de 673 hogares, cuya población incluye un marco de muestreo de 510 segmentos de conglomerados de viviendas, denominados "sectores censales". El marco de muestreo de hogares está diseñado con base a la información del Censo de Población y Vivienda de 2010 y se enfoca en el área rural y en familias que evidencian una relación con el bosque. Por lo tanto, el universo de estudio abarca un total de 176.362 personas, correspondiente al $99 \%$ de la población total de la provincia, y distribuidas en 43.032 hogares. Según el censo de 2010, la población total de la provincia de Sucumbíos para ese año fue de 176.472 personas distribuidas en un total de 43.056 hogares.

En cuanto a los registros administrativos de superficies de bosques del Ministerio del Ambiente, las superficies de bosques (nativos y deforestados) están disponibles a nivel provincial para todo el país.

Análisis de datos. Los análisis estadísticos descriptivos muestran datos con respecto al número de hogares ecuatorianos que consumen leña frente al uso de otros combustibles, y su evolución durante los últimos 35 años.

La información de consumo de leña se analizó en conjunto con la información de pobreza. El cálculo del índice de pobreza se obtuvo mediante el método de las necesidades básicas insatisfechas (NBI), que es aceptado oficialmente por el Gobierno del Ecuador. El método es descrito y aplicado por CEPAL (2001). Constituye una medida dicotómica de satisfacción de la necesidad (si se satisface o no se satisface), que considera cinco indicadores compuestos para categorizar en estratos de pobreza a la población (cuadro 1): 
Cuadro 1. Descripción de la pobreza según necesidades básicas insatisfechas (NBI).

Description of poverty by UBN.

\begin{tabular}{|c|c|c|}
\hline Necesidad básica insatisfecha & Condición & Variables censales \\
\hline 1. Acceso a vivienda & $\begin{array}{l}\text { Piso de tierra u otros materiales y material } \\
\text { precario en las paredes (caña, madera, estera, u } \\
\text { otros). }\end{array}$ & $\begin{array}{l}\text { Material de las paredes exteriores. } \\
\text { Material de los pisos. }\end{array}$ \\
\hline 2. Acceso a servicios básicos & $\begin{array}{l}\text { Sin agua por acueducto (proveyéndose el hogar } \\
\text { de agua de río, carro tanque o de lluvia) o sin } \\
\text { conexión de sanitario a alcantarillado o a pozo } \\
\text { séptico. }\end{array}$ & $\begin{array}{l}\text { Conexión con servicios públicos. } \\
\text { Tipo de sanitario. } \\
\text { Fuente de agua para el consumo humano. }\end{array}$ \\
\hline 3. Capacidad económica & $\begin{array}{l}\text { Hogares cuyo jefe(a) tenga un nivel educativo } \\
\text { menor o igual a } 2 \text { años y tres o más personas } \\
\text { por cada persona ocupada en el hogar. }\end{array}$ & $\begin{array}{l}\text { Relación o parentesco con el jefe(a) del hogar. } \\
\text { Nivel educativo y grados aprobados. } \\
\text { Actividad principal. }\end{array}$ \\
\hline 4. Acceso a educación básica & $\begin{array}{l}\text { Hogares en los cuales algún niño entre } 6 \text { y } 12 \\
\text { años de edad, pariente del jefe(a), no asista a } \\
\text { algún establecimiento educativo. }\end{array}$ & $\begin{array}{l}\text { Edad. } \\
\text { Relación o parentesco con el jefe(a) del hogar. } \\
\text { Asistencia escolar. }\end{array}$ \\
\hline 5. Hacinamiento & $\begin{array}{l}\text { Hogares con un número de personas por cuarto } \\
\text { superior a tres. }\end{array}$ & $\begin{array}{l}\text { Número de cuartos que utiliza el hogar. } \\
\text { Número de personas residentes del hogar. }\end{array}$ \\
\hline
\end{tabular}

Fuente: CEPAL (2001)

Este índice puede tomar los valores de 0 a 5 , y la línea de pobreza es el valor de 1 (incluyéndose este). Las personas se categorizan en tres niveles socioeconómicos: los no pobres (con 0 necesidades), los pobres no indigentes (si tienen 1 necesidad en cualquiera de los cinco indicadores) y pobres indigentes (si tienen 2, 3, 4, o 5 en cualquiera de las condiciones descritas anteriormente). Con los censos y encuestas oficiales del Ecuador se calcularon cifras interanualmente comparables de la evolución de la pobreza para los últimos 35 años.

La variable de consumo de leña para cocinar se construyó con la información de FAO (2012), midiendo el peso de la leña y el carbón vegetal en kilogramos, y para una frecuencia de consumo anual. Esta información se asoció con los reportes de las especies que se cortan y recolectan para leña, obtenidos del mismo estudio de Sucumbíos. Al identificar la especie de árbol se puede determinar la densidad forestal a la que le corresponde (dada en $\mathrm{kg} \mathrm{m}^{-3}$ ); esto, con el fin de obtener el volumen de consumo de leña categorizado por especie forestal, al dividir el peso por la densidad.

Una vez construida la variable de consumo, se caracterizó a las familias que consumen leña con modelos factoriales de correspondencias múltiples a partir de variables socioeconómicas y demográficas, construyendo grupos para analizar el consumo. El objeto de los modelos factoriales de correspondencias es analizar y describir, desde un punto de vista gráfico, a través del plano cartesiano, cuáles categorías de una variable $X$ se asocian o correlacionan (o son independientes) con las categorías de otra variable $Y$. De esta forma se obtienen tipos o grupos de individuos. $\mathrm{Su}$ aplicabilidad se expone en las tablas de correspondencias: si se relacionan dos variables categóricas (variables cualitativas o de atributos) se habla de correspondencia simple y, si es un número mayor, se dice que es un análisis de correspondencia múltiple.

El proceso de asignación de diferentes grados de consumo dentro de los hogares, de acuerdo al tamaño de las familias, se validó a través del coeficiente de variación y del modelo de análisis de la varianza (ANOVA).

El coeficiente de variación está definido por el cociente entre la desviación estándar y la media, cuya relación mide la magnitud de la dispersión de los datos. Un criterio comúnmente empleado define a una población como homogénea si este estadístico es menor al $20 \%$, caso contrario es heterogénea. Este coeficiente se empleó para determinar el grado de homogeneidad de los estratos sociodemográficos estudiados, así como el grado de heterogeneidad de la población a nivel global. Se analizó la distribución de este índice para definir estratos sociodemográficos, con el criterio de que un estrato es una agrupación homogénea de elementos y heterogéneo comparado con cualquier otro, de modo que, en el caso de los estratos sociodemográficos, la homogeneidad es una característica deseable.

Los grupos determinados con sus puntos de corte (rango de número de miembros por hogar asignado a cada grupo), y el análisis del grado de homogeneidad, se identificaron con respecto al tamaño de la familia. De esta forma se asegura que la variabilidad de un grupo sea igual o por lo menos no distinta respecto de los otros grupos. Esto permite proyectar la línea media de un grupo sobre otro, asegurando además que el experimento estadístico sea llevado a cabo en las mejores condiciones posibles de homogeneidad, para controlar y eliminar ciertos efectos que podrían alterar los resultados, por la introducción de sesgos o errores. 
Por otro lado, se realizaron pruebas de hipótesis con el modelo de análisis de la varianza (ANOVA), que compara la distribución del índice de condiciones sociodemográficas con los distintos grados de consumo dentro de los hogares. El objetivo principal de estas pruebas es establecer si existen o no diferencias entre los grupos de consumo; es decir, verificar la heterogeneidad del índice de condiciones sociodemográficas entre estratos. Las hipótesis aplicadas fueron las siguientes:

- Hipótesis nula $\left(\mathrm{H}_{0}\right)$ : todas las medias de los estratos son iguales, lo que es equivalente a decir que no hay diferencias entre los grupos.

- Hipótesis alternativa $\left(\mathrm{H}_{1}\right)$ : no todas las medias de los estratos son iguales, es decir, existen diferencias entre uno o más grupos.

Esta prueba se decide con la llamada probabilidad de significación estadística, teniendo que si esta probabilidad es menor que 0,05 (si se considera el $95 \%$ de confiabilidad), entonces hay diferencias significativas, caso contrario no hay diferencias entre grupos (Carrasco 2014).

Con la cantidad de leña obtenida a partir de la información del estudio de evaluación forestal de la FAO (2012) se proyectaron las medias de consumo en la información de censos y encuestas del INEC, según los tipos de familias identificados. Para medir la incidencia de este consumo en la pérdida de bosques se dividió el volumen promedio de leña consumido entre los años 2008-2014 (correspondiente a un periodo similar al reportado en el histórico de deforestación), con respecto al volumen estimado total de bosque en $\mathrm{m}^{3}$. Para obtener las hectáreas deforestadas por consumo de leña se estableció una relación de linealidad entre los promedios ponderados de bosque y leña con la deforestación neta anual. El promedio ponderado de leña $\left(2,19 \mathrm{~m}^{3} \mathrm{ha}^{-1}\right)$ se obtuvo dividiendo el volumen total de leña con respecto a la superficie total de bosque.

Conforme las cifras de deforestación a nivel provincial, se determinaron las áreas geográficas donde se registra un mayor y menor consumo de leña en términos de volumen.

\section{RESULTADOS}

Análisis descriptivo. En Ecuador, el GLP se ha consolidado como el combustible más importante para la cocción de alimentos. Del total de hogares registrados en el año 2010 (3.810.548), se determinó que un 90,7 \% utilizó gas, mientras que solo un $6,8 \%$ utilizó leña. A la fecha, esta tendencia no ha cambiado; en 2017, de un total de 4.537.839 hogares, el $92 \%$ consumió gas frente a un $4,4 \%$ que consumió leña. No obstante, la mayor parte de hogares que utilizaron leña en 2010 y 2017 corresponde al área rural (241.292 y 168.279 hogares, respectivamente).

La distribución territorial del consumo de leña determina que las provincias donde se consume el mayor vo- lumen son de la Amazonía (Morona Santiago $17.364 \mathrm{~m}^{3}$, Sucumbíos $11.867 \mathrm{~m}^{3}$ y Orellana $6.764 \mathrm{~m}^{3}$ y de la Costa (El Oro $13.747 \mathrm{~m}^{3}$ y Esmeraldas $11.998 \mathrm{~m}^{3}$. En la región interandina, en cambio, es donde se consume el menor volumen del recurso (Cañar $800 \mathrm{~m}^{3}$, Imbabura $539 \mathrm{~m}^{3}$, Carchi $162 \mathrm{~m}^{3}$ y Chimborazo $92 \mathrm{~m}^{3}$. El mayor volumen de leña consumido se registra precisamente en las provincias con mayor número de hectáreas de bosque deforestadas (Morona Santiago 7.925 ha, El Oro 6.274 ha, Esmeraldas 5.476 ha).

Índice de pobreza y su relación con el consumo de leña. La pobreza total está dada por la suma de las categorías de pobres no indigentes y pobres indigentes. En el Ecuador, la pobreza total para 1982 fue del $82 \%$, para 2007 fue del $48 \%$ y para 2017 terminó en un $39 \%$. Con una relación simple entre pobreza por NBI y combustible utilizado por los hogares, se encontró que para 2017, de los 197.497 hogares que consumieron leña, que representan un $4,4 \%$ en el consumo total de combustibles del Ecuador, el $77 \%$ pertenece a familias que son pobres y el $23 \%$ a las que no son pobres. Debido a la pobreza, la leña es el segundo combustible que usan los hogares ecuatorianos, pero es el segundo siguiendo desde muy lejos al $92 \%$ de hogares que utilizaron gas, de los cuales solo el $29 \%$ son pobres. Desde 1982 hasta la actualidad, la mayor incidencia en el consumo de esta fuente primaria de energía se ubica en hogares más pobres (figura 1).

La pobreza total se concentra en las zonas rurales del país: del total de hogares que consumieron leña en 2017 , y

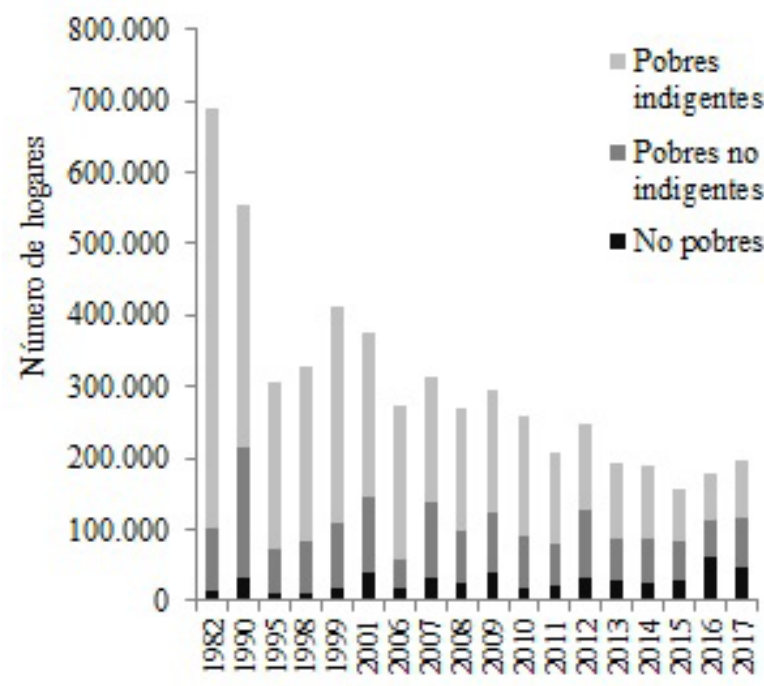

Años

Figura 1. Evolución y distribución de hogares que consumen leña con respecto a la situación de pobreza. Fuente: INEC (1982-2017).

Evolution and distribution of households that consume firewood regarding poverty. 
que se encuentran en la zona rural, el $84,7 \%$ pertenecen a familias pobres $y$, del resto de hogares ubicados en la zona urbana, tan solo el 31,5\% pertenecen a familias pobres.

Caracterización de las familias. Considerando las condiciones sociales de la familia con relación a la condición de pobreza, el acceso a servicios básicos (fuente de agua, luz y acceso a la vivienda) y el aspecto sociodemográfico (tamaño de las familias), se determinó que en aquellas viviendas con servicios básicos inadecuados y que generalmente se ubican en las zonas rurales, el consumo de leña es alto respecto a otros hogares con mejores condiciones de vida. Los resultados evidencian que los mayores índices de consumo de leña se encuentran en familias cuyas condiciones de vida no son las mejores y que se hallan dentro de la población en situación de pobreza (pobres no indigentes y pobres indigentes) (figura 2).

Modelo de economías de escala dentro del hogar con consumos marginales decrecientes. Este modelo, a diferencia de los esquemas habituales de medición, parte del supuesto de que el consumo de leña no es lineal, dado que crece a medida que aumenta el tamaño del hogar hasta un punto en que dicho crecimiento se detiene; es decir, asume economías de escala en el consumo familiar del recurso. El cálculo de coeficientes de variación permitió encontrar que en este caso, todos los estratos sociodemográficos son homogéneos en su composición individual, exceptuando el grupo de hogares pequeños. Esta excepción no supone problema alguno en los resultados, ya que a nivel global el índice es heterogéneo, atributo deseable para demostrar que los estratos sociodemográficos son diferentes entre sí (cuadro 2).

Por la probabilidad de significación estadística $(P=0,037)$, puede concluirse que sí hay diferencias significativas entre los estratos; es decir, que se rechaza la hipótesis nula y se acepta la hipótesis alternativa, ya que los datos tomados y sistematizados en la experimentación presentaron diferencias significativas entre todos los grupos $(P<0,05)$. La prueba de homogeneidad de varianzas, según el estadístico de Levene, indicó que las varianzas no son iguales $(P<0,05)$. Entonces, las medias son diferentes, lo que implica que existen diferentes medias de consumo conforme un determinado tipo de hogar (cuadro 3).

Con el modelo de economías de escala dentro del hogar se verificó que el consumo de leña no es lineal, en el sentido de que no crece proporcionalmente a medida que aumenta el tamaño del hogar; en realidad el crecimiento

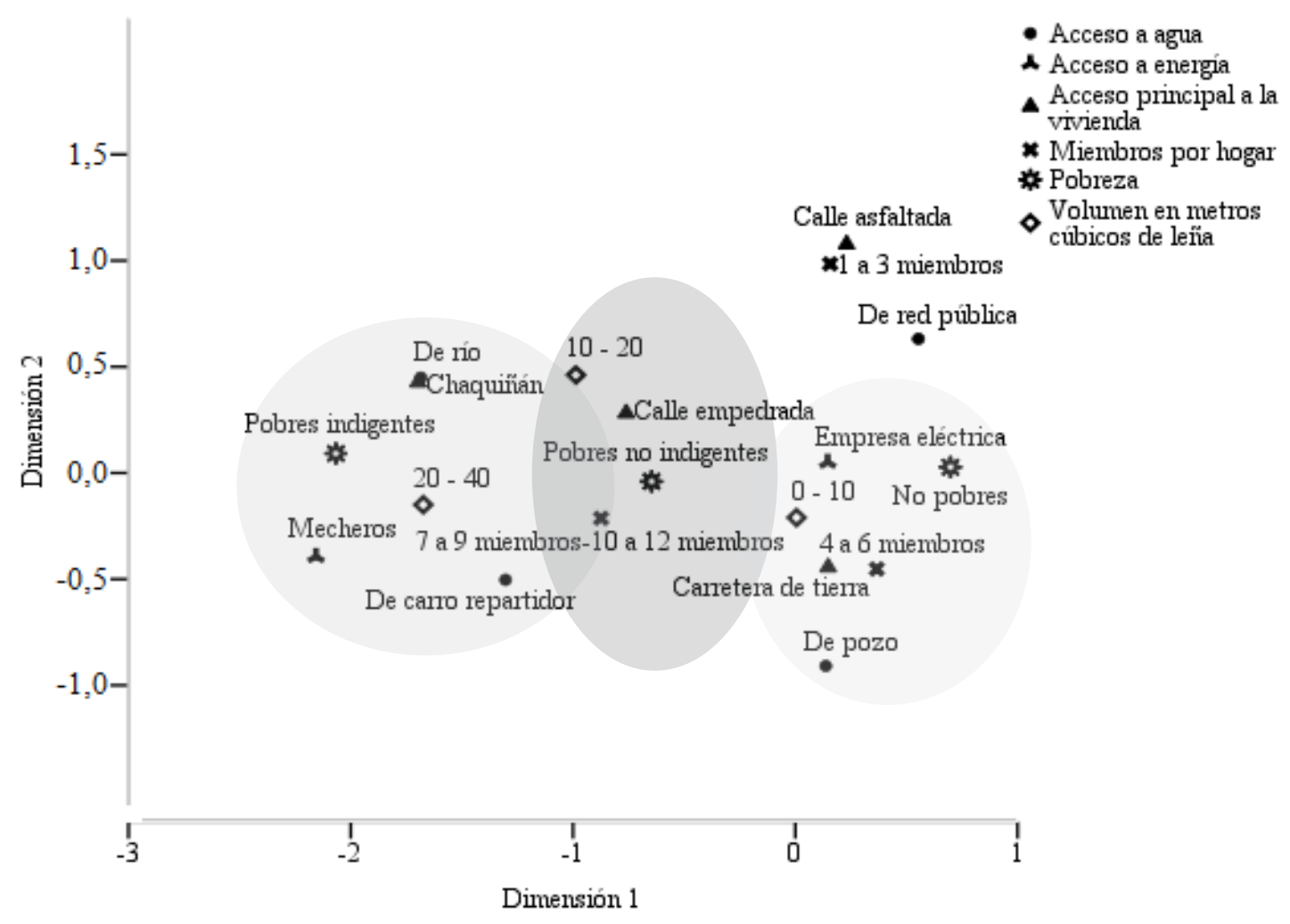

Figura 2. Modelo de correspondencias múltiples. Diagrama de conjunto de puntos de categorías: volumen de leña para cocinar con respecto al tamaño de la familia, situación de pobreza, fuentes de energía, agua y vía de acceso a la vivienda. Fuente: FAO (2012).

Multiple correspondence model. Joint plot of category points: volume of firewood for cooking regarding family size, poverty situation, energy and water sources and access to housing. 
Cuadro 2. Definición de estratos sociodemográficos y su grado de homogeneidad.

Definition of sociodemographic strata and their homogeneity degree.

\begin{tabular}{|c|c|c|c|c|c|}
\hline \multirow{2}{*}{\multicolumn{2}{|c|}{$\begin{array}{l}\text { Estratos índice de condiciones } \\
\text { sociodemográfico de los hogares }\end{array}$}} & \multirow{3}{*}{$\begin{array}{c}\text { Distribución de } \\
\text { personas (\%) }\end{array}$} & \multicolumn{2}{|c|}{ Estadísticos descriptivos del índice } & \multirow{3}{*}{$\begin{array}{c}\text { Coeficiente de } \\
\text { variación }(\%)\end{array}$} \\
\hline & & & \multirow{2}{*}{$\begin{array}{r}\text { Media } \\
2,5\end{array}$} & \multirow{2}{*}{$\begin{array}{c}\text { Desviación estándar } \\
0,655\end{array}$} & \\
\hline $1-3$ & Hogares pequeños & & & & \\
\hline $4-6$ & Hogares medianamente pequeños & 48,14 & 4,89 & 0,815 & 16,67 \\
\hline $7-9$ & Hogares medianamente grandes & 19,02 & 7,84 & 0,811 & 10,34 \\
\hline $10-12$ & Hogares grandes & 4,01 & 10,56 & 0,698 & 6,61 \\
\hline \multirow[t]{2}{*}{$\begin{array}{l}13 \text { y más } \\
\text { miembros }\end{array}$} & Hogares extremadamente grandes & 0,30 & 13,5 & 0,707 & 5,24 \\
\hline & Total & 100 & 1,9941 & 0,8147 & 40,85 \\
\hline
\end{tabular}

Fuente: FAO (2012).

Cuadro 3. Definición del consumo de leña según el tamaño del hogar.

Definition of firewood consumption according to household size.

\begin{tabular}{|c|c|c|c|c|c|c|c|c|c|}
\hline \multirow{2}{*}{$\begin{array}{l}\text { Grupo de } \\
\text { miembros } \\
\text { por hogar }\end{array}$} & \multirow{2}{*}{$\begin{array}{l}\text { Número de } \\
\text { hogares }\end{array}$} & \multirow{2}{*}{ Media $^{a}$} & \multirow{2}{*}{$\begin{array}{l}\text { Desviación } \\
\text { estándar }\end{array}$} & \multirow{2}{*}{$\begin{array}{l}\text { Error } \\
\text { típico }\end{array}$} & \multicolumn{2}{|c|}{$\begin{array}{l}\text { Intervalo de confianza } \\
\text { del } 95 \% \text { para la media }\end{array}$} & \multirow{2}{*}{ Mínimo } & \multirow{2}{*}{ Máximo } & \multirow{2}{*}{$\begin{array}{c}\text { Suma volumen } \\
\text { total de leña } \\
\mathrm{m}^{3}\end{array}$} \\
\hline & & & & & $\begin{array}{l}\text { Límite } \\
\text { inferior }\end{array}$ & $\begin{array}{l}\text { Límite } \\
\text { superior }\end{array}$ & & & \\
\hline 1 a 3 & 105 & 6,7847 & 14,5215 & 1,4172 & 3,9744 & 9,5950 & 0,001 & 121,269 & 712,395 \\
\hline 4 a 6 & 200 & 5,1239 & 12,1539 & 0,8594 & 3,4292 & 6,8186 & 0,001 & 104,840 & $1.024,772$ \\
\hline 7 a 9 & 91 & 10,8749 & 26,3907 & 2,7665 & 5,3787 & 16,3710 & 0,003 & 170,875 & 989,612 \\
\hline 10 a 12 & 20 & 14,2269 & 23,3697 & 5,2256 & 3,2896 & 25,1643 & 0,041 & 87,109 & 284,539 \\
\hline 13 y más & 1 & 3,9344 & . & . & . & . & 3,934 & 3,934 & 3,934 \\
\hline Total & 417 & 7,2308 & 17,5063 & 0,8573 & 5,5457 & 8,9160 & 0,001 & 170,875 & $3.015,253$ \\
\hline
\end{tabular}

a Prueba ANOVA significancia estadística $P=0,037$ y prueba de Levene significancia estadística $P=0,001$ *

Fuente: FAO (2012).

del consumo llega hasta un punto en que se detiene. En economía esto supone un decrecimiento de la utilidad marginal asociada al consumo de un bien o servicio, en la medida en que las necesidades son satisfechas. Sucede que la utilidad marginal (que el agente obtiene de consumir la última unidad adicional de leña) puede empezar siendo creciente, hasta llegar a un punto en que se estabiliza o incluso decrece. Se trata de la llegada a un punto de inflexión en el que cada nuevo miembro del hogar contribuye en menor medida que el anterior a incrementar el valor total de la utilidad que genera el consumo de leña, es decir, aportará utilidad de forma decreciente. Además, a partir de una cantidad arbitrariamente alta de leña, la utilidad de un metro cúbico adicional de leña podrá llegar a ser negativa.

Este hallazgo indica que, para un tamaño considerable del hogar, sobre los trece miembros, el consumo de leña deja de incrementarse o aumenta a una tasa decreciente. Así se determinó que, un hogar de uno a tres miembros consu- me anualmente en promedio $6,78 \mathrm{~m}^{3}$ de leña, un hogar de cuatro a seis miembros $5,12 \mathrm{~m}^{3}$, una familia de siete a nueve miembros $10,87 \mathrm{~m}^{3}$. De diez a doce miembros, el consumo se registra en $14,23 \mathrm{~m}^{3}$ de leña. De trece miembros en adelante el consumo tiende a decrecer a $3,9 \mathrm{~m}^{3}$ de leña, es decir, que al incrementarse considerablemente el número de miembros en el hogar el consumo no se incrementa.

Distribución del consumo por condiciones socioeconómicas. Estos resultados se pueden asociar con la hipótesis de la curva ambiental de Kuznets (Grossman y Krueger 1995, Stern et al. 1996), la misma que muestra una relación que tiene la forma de una $U$ invertida, entre los ingresos percápita y el impacto ambiental asociado a la trayectoria de crecimiento económico del territorio bajo análisis. Esta curva indica que bajos niveles de ingresos están asociados a un deterioro ambiental. Sin embargo, los ingresos podrán llegar a cierto nivel que será suficiente para que esta 
economía pueda invertir en calidad ambiental y, por ende, esté en capacidad de mejorar sus condiciones ambientales o reducir sus impactos. En el caso que nos concierne en este artículo, a partir del modelo de correspondencias múltiples se verifica que desde 1982 existe una tendencia monótona (no lineal) creciente en el consumo de GLP, a medida que mejoran las condiciones de vida de las familias o específicamente su riqueza real. Este combustible tiene ciertas ventajas ambientales y sociales (FAO 2006b, Lagunes-Díaz et al. 2015). De acuerdo al Panel Intergubernamental de Cambio Climático (IPCC 2006), la madera tiene un factor de emisión promedio de $112 \mathrm{Mg}$ de $\mathrm{CO}_{2} / \mathrm{TJ}$, casi el doble que otros combustibles empleados para la cocción de alimentos, como el GLP (63,1 Mg de $\left.\mathrm{CO}_{2} / \mathrm{TJ}\right)$, o la gasolina $\left(69,1 \mathrm{Mg}\right.$ de $\left.\mathrm{CO}_{2} / \mathrm{TJ}\right)$. Asimismo, el GLP no emite material particulado como la leña, que puede ser nocivo para la salud. Desde esta perspectiva, el Ecuador se encontraría en la fase creciente de la curva ambiental de Kuznets, en donde las condiciones de pobreza se asocian al consumo de fuentes energéticas más contaminantes (figura 3 ).

Estimación del volumen de consumo de leña. Con base al censo poblacional de 2010, un total de 3.815.246 hogares consumieron un volumen agregado de leña de 24.625.569 $\mathrm{m}^{3}$ al año y, de acuerdo a la encuesta de empleo de 2017,
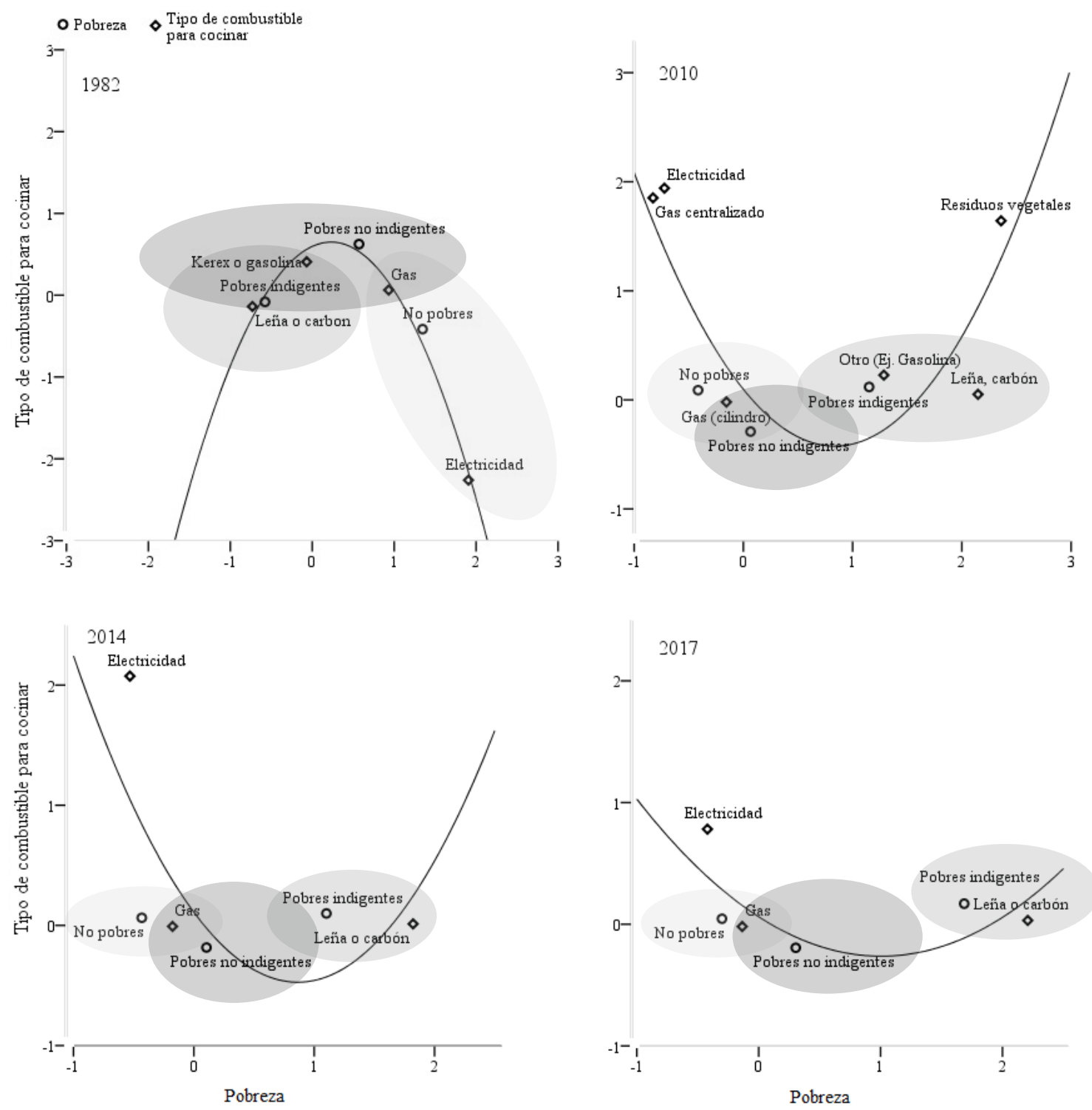

Figura 3. Modelo de correspondencias simple para testear la hipótesis de Kuznets ambiental: relación entre el tipo de combustible utilizado para cocinar y el nivel de pobreza para los años 1982, 2010, 2014 y 2017. Fuente: INEC (1982, 2010, 2014, 2017).

Simple correspondence model to test the environmental Kuznets hypothesis: relationship between the type of fuel used for cooking and the level of poverty for the years 1982, 2010, 2014 and 2017. 
4.522.603 hogares consumieron $28.805 .987 \mathrm{~m}^{3}$ al año. El volumen consumido no ha variado en los últimos cuatro años y tampoco considerablemente en los últimos 10 años. El volumen promedio de leña conforme a los datos históricos de deforestación (2008-2014) se estimó en 24.898.075 $\mathrm{m}^{3}$ al año.

A partir de datos del consumo de leña, y según la especie forestal empleada, es posible definir que un total de $521.804 .654 \mathrm{~m}^{3}$ de madera serían destinados al uso de leña (MAE 2015). Se estima que el consumo de este recurso para fines productivos o comerciales sería de $496.906 .579 \mathrm{~m}^{3}$; es decir, que tan solo el $5 \%$ del uso de leña es destinado a un consumo doméstico y un $95 \%$ a un consumo comercial o productivo (de restaurantes dedicados a la venta de pollos a la brasa, asaderos, ladrilleras, panaderías, criaderos de pollos, entre otros). Cabe anotar que el registro del consumo de leña en los establecimientos informales seguramente será más elevado, aunque no se dispone de esta información.

Incidencia del consumo de leña en los bosques. Conforme datos arrojados por el estudio de FAO (2012), se estima que, en la provincia de Sucumbíos, el $99 \%$ de los hogares cuyas viviendas se encuentran con presencia de bosques, utilizan leña proveniente de especies nativas, principalmente de la guaba o guabillo (Inga striata Benth), alrededor de 7,23 $\mathrm{m}^{3}$ por familia al año y, en promedio nacional se calculó $6,37 \mathrm{~m}^{3}$ por familia al año, cerca de $4 \mathrm{Mg}$ por familia.

Al tomar el volumen promedio de leña del periodo 2008-2014 asociado a la información del volumen total de bosque estimado $\left(\mathrm{m}^{3}\right)$, se obtuvo que el volumen de leña consumido en ese periodo incide sobre los bosques en un $1 \%$; es decir que anualmente se deforestan 430,6 ha de bosque por consumo de leña para subsistencia, a diferencia de $8.593,8$ ha que se estima se estarían deforestando anualmente para usos comerciales o productivos de leña.

\section{DISCUSIÓN}

El propósito de esta investigación ha sido analizar si son los más pobres el origen de la presión ambiental sobre los bosques nativos del Ecuador, debido a su consumo de leña; es decir, se ha evaluado la pobreza como determinante del consumo doméstico de leña y el efecto que esta condición ha generado en la deforestación en este territorio.

El problema de la deforestación en el Ecuador es complejo por varias razones. Son múltiples las causas que lo originan y múltiples los actores involucrados (Wunder 2000, Añazco et al. 2010); por lo tanto, existen altos conflictos de intereses en su manejo. No obstante, la información para el análisis y la definición de estrategias de gestión integral es escasa y dispersa. En estas condiciones, a pesar de que la mayor causa de la deforestación en los bosques ecuatorianos suele asociarse a la expansión de la frontera agropecuaria (MAE 2014), se propone aportar en este artículo con el estudio de la deforestación originada en el consumo de leña. Esta dimensión de la deforestación ha sido escasamente abordada en la literatura, y las estimaciones existentes son inexactas al asumir una estructura lineal en el consumo de los hogares (CESA 1992, Proaño 2005). Además, como lo corrobora el presente análisis, existe una fuerte dependencia de los hogares pobres respecto de este servicio ecosistémico, que amerita una estrategia de gestión específica para estas poblaciones.

Con este objetivo, se calculó el volumen de consumo de leña que se realiza a nivel nacional, tomando como punto de partida la hipótesis de un consumo marginalmente decreciente, de acuerdo al tamaño del hogar, que muestra una suerte de economías de escala en el uso doméstico de esta fuente de energía, cuya utilización predomina entre hogares en condiciones de pobreza; es decir, los mayores índices de consumo de leña se encuentran en hogares con altos niveles de pobreza y cuyas condiciones de vida no son las mejores. Sin embargo, las necesidades básicas insatisfechas de los hogares no serían el único determinante de los procesos de deforestación que se originan en el consumo de leña. En este trabajo se demuestra que la parte más importante del consumo de esta fuente de energía no se produce en el autoconsumo para subsistencia de los más pobres sino en el consumo para negocios o actividades productivas. En efecto, se calcula que apenas el $5 \%$ del consumo total de leña se emplea para cocinar en los hogares y estas actividades explican apenas el $1 \%$ de la deforestación en el territorio nacional.

Este trabajo contribuye desde un punto de vista metodológico y también práctico a la gestión de los recursos forestales en el Ecuador. Metodológicamente, a su vez, también es doble la contribución que se realiza. Por un lado, se demuestra de manera consistente que, por condiciones sociodemográficas, existen estratos de consumo heterogéneos que no realizan de forma lineal el consumo doméstico de leña, ya que a medida que se incrementa el tamaño del hogar sobre los trece miembros, el consumo deja de aumentar o crece a un ritmo decreciente. Al suponer un esquema lineal de consumo en los hogares, directamente se asume que todas las familias poseen las mismas características socioeconómicas y demográficas, un supuesto bastante irreal en la práctica. Por esta razón, los presentes resultados definen una distancia importante con aquellos estudios que asumen un consumo lineal entre hogares, como es el caso de CESA (1992), cuyos resultados sobreestiman el consumo de leña en un rango de entre 12,5 y $14,5 \mathrm{~m}^{3}$ por familia al año en la provincia de Tunguragua, que es prácticamente el doble del consumo promedio que se obtiene en este artículo para esa misma provincia $\left(6,28 \mathrm{~m}^{3}\right)$.

El consumo de leña en el Ecuador para una familia promedio de cuatro personas sería de aproximadamente $0,018 \mathrm{~m}^{3}$ de leña al día. Se considera que es una estimación apropiada si se compara con el cálculo de Ramírez y Taborda (2014) para una población campesina de la región colombiana de Antioquia, quienes obtienen a través de un 
cálculo lineal, que pudiera contener una sobreestimación, un consumo 2,5 veces superior, que serviría para mantener fuego encendido durante un lapso de casi 8 horas al día. Otros elementos de contraste son las cifras de Hoffman (1982) para Chile (de 4 a $6 \mathrm{Mg}$ por familia) o el estudio de Shaheen et al. (2016), que determina para la región de Cachemira en el Himalaya, un consumo de leña por hogar al año de $16,2 \mathrm{Mg}$ (5,9 por día percápita), un volumen muy alto si se compara con los resultados del presente estudio, pero que se explica por las diferentes necesidades energéticas y condiciones de vida en esos territorios. En particular, la condición climática tendrá un peso fundamental en sus cifras, aspecto que, de acuerdo a los hallazgos de este trabajo, no constituye un determinante del consumo de leña ni fuente de deforestación significativa en la región interandina ecuatoriana. A escala nacional, estos resultados determinan una deforestación asociada a este consumo, que se estima en 430,6 ha de bosque.

La segunda contribución metodológica del trabajo es que sirve para complementar las cuentas biofísicas del consumo de biomasa en el Ecuador, en base a las cuales se definen estrategias para la conservación del ambiente (MAE 2019). Esto es particularmente relevante para aquellos territorios con hogares en serias condiciones de pobreza, los cuales muestran una significativa dependencia respecto del uso de esta fuente de energía, en donde la ampliación de subsidios para el uso de otras fuentes energéticas como el GLP puede debatirse desde argumentos ambientales, aunque contraríe el rumbo de la estrategia de eliminación progresiva de subsidios a diversos tipos de combustibles, que por efecto de la crisis económica actual de este país se está definiendo. En efecto, especies nativas de madera como el Inga striata, un árbol de guabo, se hallan actualmente bajo presión por el uso doméstico como combustible de madera; sin embargo, su utilización responde fundamentalmente a la cobertura de necesidades energéticas básicas de los hogares pobres, por lo que una estrategia orientada a promover la sustitución de fuentes energéticas será más viable para estas poblaciones.

A partir de los resultados obtenidos con la aplicación del modelo de correspondencias múltiples, se puede interpretar que a medida que mejoran las condiciones de vida de las familias, o específicamente, su riqueza real, los hogares tienden a consumir combustibles más limpios con menos emisiones de GEI, como es el caso del GLP. Se trataría de un efecto tipo "Kuznets ambiental", que mostraría que mientras menos factible sea mejorar las condiciones de vida de las familias, estas tenderán a mantener o incrementar el consumo de leña, contribuyendo a la degradación ambiental en términos de deforestación.

En el presente trabajo se argumenta que la población que sufre de privaciones, sufre también de desigualdad social, desigualdad que alude a la falta de acceso a servicios y satisfacción de necesidades básicas, planteando a la desigualdad social en este caso específico, no basada en la distribución de la renta entre los ciudadanos, sino ligada al goce de derechos fundamentales como acceso a vivienda, agua, energía, educación, etc. A medida que los hogares mejoran sus condiciones de vida, tienden a utilizar otro tipo de combustible distinto a la leña. Esta tendencia monótona no se ha modificado desde 1982. Simplemente estas poblaciones consumen leña porque es un recurso barato a su alcance, y quizá incluso un "mejor satisfactor" de necesidades, por ejemplo, para calentarse o cocinar.

No sucede lo mismo con el uso comercial o productivo de la leña. Se calcula que, en el Ecuador, la leña está entre los principales tipos de uso de los árboles, con un $19 \%$ de participación (MAE 2015). La distribución territorial de su consumo permite identificar la mayor parte de la deforestación en varias provincias amazónicas y de la costa, siendo mucho menos marcada la deforestación por consumo de leña en la región interandina. En efecto, la leña como fuente de calor sería apenas empleada en la sierra ecuatoriana, por lo que la deforestación en la costa y Amazonía podría ser directamente asociada al consumo comercial y productivo del recurso; $y$, por lo tanto, también a la pérdida intensiva de biodiversidad en estas regiones.

Con estos elementos se contribuye a la cuantificación de la deforestación asociada al uso de un servicio ecosistémico fundamental para la supervivencia de territorios en condiciones de pobreza. Sin embargo, para futuras investigaciones se considera importante recomendar una actualización del modelo, con una fuente de datos más reciente, dado que las condiciones sociales son dinámicas. El modelo desarrollado en este artículo se basa en la encuesta de la FAO, del estudio piloto realizado en la provincia de Sucumbíos en el año 2012, que en la actualidad presenta un sesgo de inclusión de unidades de muestreo por el crecimiento de la población. No obstante, los resultados que aquí se presentan son consistentes porque la estimación de consumo realizada se ha proyectado a las bases de datos de los últimos 10 años del INEC, por ser relativamente actuales, representativas y confiables a nivel de las provincias.

Con fuentes de informaciones completas y actualizadas, se propone que en futuros estudios se considere estimar el consumo de leña doméstico y comercial, construyendo un modelo estadístico para cada uno, ya que la dinámica comercial no es la misma que la de las familias que tienen consumos no lineales. Realizar pruebas y ensayos de verificación hasta que el nivel de significancia de las pruebas estadísticas avale los experimentos diseñados.

\section{CONCLUSIONES}

Entre los hogares más pobres existe una fuerte dependencia respecto del consumo de leña, observada a partir de la aplicación de un modelo de economías de escala, que asume un consumo marginal decreciente de leña en los hogares ecuatorianos porque existen estratos de consumo heterogéneos que no asocian un consumo significativamente más elevado para las familias más numerosas. Los 
resultados son particularmente relevantes para el planteamiento de políticas públicas específicas para estos sectores, porque existen argumentos ambientales que pueden servir para el debate sobre la ampliación de subsidios para el uso de fuentes energéticas alternativas a la leña en el autoconsumo. No obstante, el mayor reto en una estrategia ambiental por la deforestación originada en el consumo de leña, deberá centrarse en la demanda que realizan diversos negocios y actividades productivas, que concentran la mayor parte del consumo de esta fuente de energía, y que, por lo tanto, atenúan el rol de los más pobres (o incluso los desvincula) de la presión ambiental en los bosques nativos del Ecuador.

\section{AGRADECIMIENTOS}

El primer autor agradece a la Facultad Latinoamericana de Ciencias Sociales (FLACSO Ecuador) por la acogida en la institución que permitió la realización de los estudios de Maestría con la investigación "La pobreza como determinante del consumo de leña para cocinar y su efecto en la deforestación de los bosques del Ecuador entre 1982-2017”.

\section{REFERENCIAS}

Añazco M, M Morales, W Palacios, E Vega, AL Cuesta. 2010. Sector Forestal Ecuatoriano: Propuestas para una gestión forestal sostenible. Quito, Ecuador. ECOBONA-Intercooperation. Serie Investigación y Sistematización No. 8. Programa Regional. 213 p.

Carrasco F. 2014. Construcción de un modelo de clasificación socioeconómica para el descuento de las colegiaturas de los estudiantes de la FLACSO. Tesis Magíster en Economía del Desarrollo. Quito, Ecuador. Departamento de Desarrollo, Ambiente y Territorio, Facultad Latinoamericana De Ciencias Sociales. 94 p.

CEPAL (Comisión Económica para América Latina y el Caribe, CL). 2001. Estudios estadísticos y prospectivos. El método de las necesidades básicas insatisfechas (NBI) y sus aplicaciones en América Latina. Santiago de Chile, Chile. CEPAL. 52 p.

CESA (Central Ecuatoriana de Servicios Agrícolas, EC). 1992. El deterioro de los bosques naturales del callejón interandino del Ecuador. Quito, Ecuador. Programa de Conservación de los Recursos Naturales en Áreas Marginales de la Sierra Ecuatoriana. 79 p.

Eurostat (European Statistical Office, IT). 2001. Economy-wide material flow accounts and derived indicators. A methodological guide. Luxemburgo, Luxemburgo. European Communities. $92 \mathrm{p}$.

FAO (Food and Agriculture Organization of the United Nations, IT). 2006a. Programas forestales nacionales: qué contribución puede hacer la FAO. Roma, Italia. Unasylva 225: 57-3.

FAO (Food and Agriculture Organization of the United Nations, IT). 2006b. Los bosques y la salud humana. Roma, Italia. Unasylva 224: 57-2.

FAO (Food and Agriculture Organization of the United Nations, IT). 2012. Encuesta socioeconómica en hogares y su relacion con el bosque. Sucumbíos, Ecuador. FAO. 18 p.
FAO (Food and Agriculture Organization of the United Nations, IT). 2017. La transición al carbón vegetal. La ecologización de la cadena de valor del carbón vegetal para mitigar el cambio climático y mejorar los medios de vida locales. Roma, Italia. FAO. 11 p.

Grossman GM, AB Krueger. 1995. Economic Growth and the Environment. The Quarterly Journal of Economics 110(2):353-377.

Hoffmann A. 1982. Flora silvestre de Chile: zona austral, una guía ilustrada para la identificación de las especies de plantas leñosas del sur de Chile. Santiago, Chile. Ediciones Fundación Claudio Gay. 257 p.

INEC (Instituto Nacional de Estadística y Censos, EC). 19822017. Censos de Población y Vivienda: 1982, 1990, 2001 y 2010; Encuestas de Condiciones de Vida (ECV): 1995, 1998, 1999, 2005-2006 y 2013-2014; Encuestas Nacionales de Empleo, Desempleo y Subempleo (ENEMDU) cuarta ronda: 2007- 2017. Quito, Ecuador. INEC.

IPCC (Intergovernmental Panel on Climate Change, IT). 2006. Guidelines for National Greenhouse Gas Inventories. Hayama, Japón. National Greenhouse Gas Inventories Programme, IGES, Eggleston HS, L Buendia, K Miwa, T Ngara, K Tanabe eds.

Lagunes-Díaz E, ME González-Ávila, A Ortega-Rubio. 2015. Transición de leña a gas licuado a presión (GLP) en el sur de México, oportunidad para la mitigación del cambio climático en la región menos desarrollada del país. Acta Universitaria 25 (6): 30-42. DOI: 10.15174/au.2015.853

MAE (Ministerio del Ambiente del Ecuador, EC). 2010. Aprovechamiento de los Recursos Forestales 2007-2009. Quito, Ecuador. Consultado 6 oct. 2017. Disponible en http://suia. ambiente.gob.ec/

MAE (Ministerio del Ambiente del Ecuador, EC). 2013. Proyecto Sistema Nacional de Control Forestal. Quito, Ecuador. Consultado 9 oct. 2017. Disponible en http://www.ambiente.gob.ec/

MAE (Ministerio del Ambiente del Ecuador, EC). 2014. Evaluación Nacional Forestal. Resultados. Quito, Ecuador. Consultado 8 en. 2018. Disponible en http://suia.ambiente.gob. $\underline{\mathrm{ec} /}$

MAE (Ministerio del Ambiente del Ecuador, EC). 2015. Línea Base de Deforestación del Ecuador Continental. Quito, Ecuador. Consultado 2 oct. 2017. Disponible en http://sociobosque.ambiente.gob.ec/

MAE (Ministerio del Ambiente del Ecuador, EC). 2019. Sistema de Contabilidad Ambiental Nacional. Quito, Ecuador. Consultado 17 ene. 2019. Disponible en http://www.ambiente. gob.ec/

McKenzie M. 1994. La política y la gestión de la energía rural: La experiencia del Ecuador. Quito, Ecuador. Facultad Latinoamericana de Ciencias Sociales (FLACSO). p. 245-283.

Proaño D. 2005. Estudio de tendencias y perspectivas del Sector Forestal en América Latina. Informe Nacional Ecuador. Roma, Italia. FAO. 55 p.

Ramírez JF, AL Taborda. 2014. Consumo de leña en fogones tradicionales en familias campesinas del oriente antioqueño. Colombia. Revista de Investigación Agraria y Ambiental 9(1): 99-114. DOI: $10.22507 /$ pml.v9n1a8

Shaheen H, B Azad, A Mushtaq, R Ahmad. 2016. Patrón de consumo de leña y sus impactos en la estructura del bosque en Cachemira, Himalaya. Bosque 37(2): 419-424. DOI: 
10.4067/S0717-92002016000200020

Sierra F, F Mejía, C Guerrero. 2011. Leña como combustible doméstico en zonas rurales de Usme. Vol. 75. Bogotá, Colombia. Informador Técnico. DOI: 10.23850/22565035.17

Stern D, M Common, E Barbier. 1996. Economic growth and environmental degradation: the enviromental Kuznets curve and sustainable development. World Development 24(7):1151-1160.

Vallejo MC. 2010. Biophysical structure of the Ecuadorian economy, foreign trade, and policy implications. Eco- logical Economics 70: 159-169. DOI: 10.1016/j.ecolecon.2010.03.006

Wunder S. 1996. Los caminos de la madera. Una investigación de los usos domésticos y comerciales de los productos de la madera y su relación con el proceso de deforestación. Quito, Ecuador. Unión Internacional para la Conservación de la Naturaleza (UICN). 422 p.

Wunder S. 2000. The Economics of Deforestation. The Example of Ecuador. Palgrave Macmillan, London. DOI: $10.1057 / 9780230596696$

Recibido: $25 / 01 / 19$

Aceptado: 27/05/19 